\title{
IMPACTO DA UTILIZAÇÃO DE PREVISÕES “DEFASADAS” NO SISTEMA DE PREVISÃO DE TEMPO POR CONJUNTO DO CPTEC/INPE
}

\author{
LÚCIA HELENA RIBAS MACHADO, ANTÔNIO MARCOS MENDONÇA, \\ RENATA WEISSMANN BORGES MENDONÇA, JOSÉ PAULO BONATTI
}

\author{
Centro de Previsão de Tempo e Estudos Climáticos / Instituto Nacional de Pesquisas Espaciais \\ (CPTEC/INPE), Cachoeira Paulista-SP, Brasil
}

lucia.ribas@cptec.inpe.br, marcos.mendonca@cptec.inpe.br, renata.mendonca@cptec.inpe.br, paulo.bonatti@cptec.inpe.br

Recebido Setembro 2008 - Aceito Outubro 2009

\begin{abstract}
RESUMO
Neste trabalho é descrita a aplicação da técnica de previsões defasadas no sistema de previsão de tempo por conjuntos do Centro de Previsão de Tempo e Estudos Climáticos (EPS-CPTEC/INPE). Os dados do CPTEC/INPE consistem em uma amostra de dois meses com previsões de 15 dias para as variáveis: altura geopotencial em $500 \mathrm{hPa}$, temperatura do ar no nível de $850 \mathrm{hPa}$, e pressão atmosférica ao nível médio do mar. O estudo consiste em investigar: 1) o desempenho do EPS-CPTEC/INPE utilizando a técnica de previsões defasadas comparado àquele do conjunto operacional; 2) a relação entre o espalhamento e o desempenho da previsão, a fim de avaliar o uso da dispersão como preditor do desempenho. Os resultados indicam que a utilização de previsões defasadas em $12 \mathrm{~h}$, melhora o desempenho do conjunto operacional, contribuindo para aumentar o espalhamento do conjunto e, conseqüentemente, reduzir a sub-dispersão do sistema. Também foi observado que o conjunto defasado tem desempenho comparável àquele do conjunto operacional e que há uma tendência de desempenho alto quando o espalhamento é baixo, para os prazos de 5 e 7 dias de previsão. Estes resultados servem como base para a implementação operacional desta técnica, que apresenta baixo custo computacional, e contribui para a utilização mais eficiente das previsões por conjunto do CPTEC/INPE.
\end{abstract}

Palavras Chave: Previsão de Tempo por Conjuntos, Espalhamento, Previsões Defasadas

\begin{abstract}
THE IMPACT OF USING LAGGED FORECASTS ON THE CPTEC/INPE ENSEMBLE PREDICTION SYSTEM

In this work we report the application of the lagged average forecasting technique to CPTEC/INPE ensemble forecast. The CPTEC/INPE data consist of two months samples of 15 days forecast for the variables: geopotential height at $500 \mathrm{hPa}$, air temperature at $850 \mathrm{hPa}$ and mean sea level atmospheric pressure. We focus on the following: 1) Does the lagged averaged ensemble forecast improve forecast skill compared to the CPTEC/INPE operational ensemble? 2) Is the dispersion of the ensemble useful in predicting forecast skill? The results indicate that the utilization of 12h-lagged average forecasts improves the performance of the operational ensemble contributing to increase the ensemble spreading and, consequently, to reduce the under-dispersion of the system. Also we observed that lagged average forecast (LAF) shows similar performance of the operational EPS-CPTEC/INPE and that there is a tendency to higher performance when spread forecast is low, for 5 and 7 day forecast. These results provide the basis for the operational implementation of the LAF technique, which has low computational cost, and contribute to a more efficient utilization of the CPTEC/INPE ensemble predictions.
\end{abstract}

Keywords: Ensemble Prediction System, Spread, Lagged Average Forecasts 


\section{INTRODUÇÃO}

As previsões meteorológicas contêm erros que são inerentes ao processo de produção de tais prognósticos. A redução/perda do desempenho dos modelos em prever os movimentos de escala sinótica é causada pelo crescimento dos erros associados a ambos: às incertezas nas condições iniciais (Lorenz, 1963) e às imperfeições no modelo (Reynolds, 1994). Previsões, partindo de condições iniciais ligeiramente distintas, podem divergir fortemente após algum tempo de integração e, não existe uma única solução para o estado futuro da atmosfera, mas um conjunto de possíveis soluções. Desta forma, a técnica de previsão de tempo por conjunto pode ser uma ferramenta útil para tentar estimar a confiabilidade das previsões uma vez que, de forma geral, pode considerar as incertezas das condições iniciais e do modelo em sua produção.

A forma mais simples de utilizar as previsões por conjunto é através do conjunto médio, que representa a melhor estimativa do estado futuro da atmosfera. A previsão do conjunto médio apresenta um desempenho melhor em relação àqueles dos membros individuais do conjunto uma vez que alguns erros individuais são cancelados na média (Murphy, 1988; Tracton e Kalnay, 1993).

A variabilidade dos membros do conjunto, ou espalhamento, pode fornecer uma medida da incerteza da previsão. Idealmente, quanto maior o espalhamento dos membros do conjunto, maior é o intervalo de soluções possíveis da atmosfera e menor é o nível de confiabilidade de uma previsão, ou seja, os valores altos/baixos de espalhamento indicam baixa/alta confiabilidade da previsão (Barker, 1991).

A confiabilidade ou incerteza de uma previsão pode ser avaliada a partir do espalhamento do conjunto (Molteni e Palmer, 1991). O espalhamento pode ser calculado em relação ao conjunto médio ou em relação à previsão de controle e seu valor é definido como sendo a distância média quadrática dos membros em relação ao conjunto médio (Buizza, 1997).

A relação entre o desempenho da previsão e o espalhamento tem sido investigada por vários autores como forma de avaliar a qualidade da previsão e indicar o limite de previsibilidade do sistema de previsão de tempo por conjunto. Segundo Hoffman e Kalnay (1983) existe uma forte relação entre a dispersão do conjunto e a perda de previsibilidade. Molteni e Palmer (1991) encontraram valores de correlação entre o espalhamento e o desempenho em termos da raiz do erro quadrático médio $(R M S)$, entre 0,3 e 0,4 , no $7^{\circ}$ dia de previsão, no Hemisfério Norte, para o campo de altura geopotencial em $500 \mathrm{hPa}$ do Sistema de Previsão de Tempo por Conjunto (EPS) do European Center for Medium-Range Weather Forecasts (ECMWF). Molteni et al. (1996) mostrou, através do diagrama de dispersão entre o $R M S$ e o espalhamento das previsões de altura geopotencial em $500 \mathrm{hPa}$, sobre a região da Europa, que a dispersão e a relação espalhamento/desempenho são maiores no verão sendo que o espalhamento está mais correlacionado com o desempenho das previsões nos prazos de 5 e 7 dias de previsão. Buizza (1997) também estudou a relação entre estas variáveis e encontrou que há $20 \%$ (45\%) de correspondência entre o espalhamento e o desempenho de previsões perturbadas no $7^{\circ}$ dia de previsão durante estações quentes (frias).

No Centro de Previsão de Tempo e Estudos Climáticos (CPTEC/INPE) o sistema de previsão de tempo por conjunto (EPS-CPTEC/INPE) foi implementado operacionalmente em outubro de 2001 (http://www.cptec.inpe.br/prevnum/ exp_ensemble.shtml), motivado pelos estudos de Coutinho (1999), cujos resultados indicaram que a aplicação do conjunto no modelo de circulação geral atmosférico do CPTEC/INPE (MCGA-CPTEC/INPE) poderia trazer benefícios para a previsão de médio prazo para diversas regiões do globo, especialmente para a América do Sul. Mendonça e Bonatti (2004), comparando o desempenho das previsões do EPSCPTEC/INPE para o período de outubro de 2001 a setembro de 2003, verificaram que o desempenho do conjunto médio é, na média do período, superior àquele da previsão de controle (determinística). Entretanto, os resultados do trabalho de Mendonça e Bonatti (2006) mostraram que a taxa de crescimento do espalhamento das previsões do EPS-CPTEC/INPE é inferior à taxa de crescimento dos erros do modelo, o que caracteriza o sistema como subdispersivo.

A utilização de previsões defasadas (LAF -"lagged average forecast" - em inglês) representa uma das primeiras tentativas de produzir previsões por conjunto, sendo esta apresentada por Hoffman e Kalnay (1983). O método (LAF) considera um conjunto composto de previsões inicializadas a partir de análises consecutivas, em que são incluídas as previsões mais recentes e aquelas iniciadas em um ou mais dias antes. Uma das vantagens deste método é o aumento do número de previsões (membros do conjunto) sem custo operacional. A desvantagem é que as previsões mais antigas podem ter desempenho inferior às previsões mais recentes. Este problema pode ser contornado pela ponderação dos diferentes membros em função dos desempenhos relativos (Dalcher et al., 1988).

O objetivo deste estudo é apresentar um esquema para utilização de previsões defasadas no EPS-CPTEC/INPE, além de avaliar o impacto da inclusão destas novas previsões no desempenho do conjunto. As principais características avaliadas são o desempenho do conjunto médio e o espalhamento do conjunto através do cálculo da raiz do erro quadrático médio, da correlação de anomalias e do desvio padrão dos membros do conjunto. É também avaliada a relação entre o espalhamento e o desempenho das previsões de tempo por conjunto, a fim de investigar o uso do espalhamento como preditor do desempenho 
do EPS-CPTEC/INPE. Na seção 2 são descritos os dados e a metodologia utilizada neste estudo. Os resultados são discutidos na seção 3, e na seção 4 são apresentadas as conclusões.

\section{DADOS E METODOLOGIA}

\subsection{Dados}

Os dados utilizados neste estudo são as condições iniciais (análises) fornecidas pelo National Centers for Environmental Predictions (NCEP) e as previsões de tempo por conjunto do CPTEC/INPE, nos horários sinóticos 0000 UTC e 1200 UTC, na resolução T126L28, para o período de 10 de julho a 10 de setembro de 2007. Para o cálculo das anomalias do campo de geopotencial foi usado a Climatologia da Reanálise 2 do NCEP (Kanamitsu et al., 2002).

\subsection{Sistema de Previsão de Tempo por Conjunto do CPTEC/INPE}

As previsões de tempo por conjunto do CPTEC/INPE são realizadas diariamente duas vezes ao dia (0000 e 1200 UTC) para um prazo de 15 dias. No total são gerados 15 membros (com as integrações do modelo partindo de diferentes condições iniciais), sendo um membro gerado a partir da condição inicial de controle (sem perturbações) e 14 a partir de condições iniciais perturbadas.

As condições iniciais perturbadas do EPS-CPTEC/INPE são geradas utilizando o método denominado "EOF-based perturbation", desenvolvido por Zhang e Krishnamurti (1999). Este método considera que, durante os primeiros dias de integração do modelo, a perturbação cresce quase linearmente com o tempo. O procedimento de geração da perturbação consiste em adicionar à análise de controle (não perturbada) pequenas perturbações aleatórias, comparáveis aos erros de previsão; integrar o modelo por 36 horas (intervalo ótimo), a partir das análises de controle e perturbadas, com saídas a cada três horas. É realizada, então, uma análise de Funções Ortogonais Empíricas (EOF) da série temporal formada pela diferença entre a previsão perturbada e a de controle para a obtenção do modo de crescimento mais rápido (considerado como o autovetor associado ao maior autovalor). É realizado um re-escalonamento destas perturbações de forma que o desvio padrão, destas perturbações, seja da ordem das perturbações iniciais. Este modo de crescimento mais rápido é somado e subtraído à condição inicial de controle para formar duas condições iniciais otimamente perturbadas.

\subsection{Experimentos com EPS-CPTEC/INPE Utilizando Previsões Defasadas}

O novo sistema de previsão de tempo por conjunto a ser testado, conjunto defasado, é representado esquematicamente na Figura 1. Na técnica empregada neste trabalho são consideradas basicamente as previsões iniciando em um determinado tempo $\mathrm{t}$ (por exemplo, 00 UTC) e as previsões defasadas em 12 horas, isto é, $\mathrm{t}-12 \mathrm{~h}$. As primeiras correspondem aos membros do EPS-CPTEC/INPE atuais e, às últimas, constituem os novos membros que serão agregados ao conjunto, aqui denominados membros defasados.

Para avaliar qual é a configuração mais adequada ao adicionar previsões defasadas ao EPS-CPTEC/INPE, seis

CONJUNTO OPERACIONAL (atual: 15 membros)

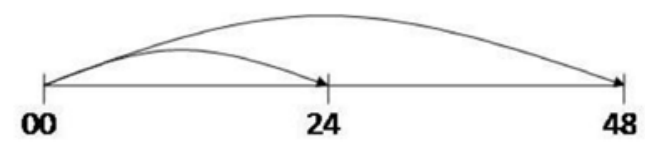

CONJUNTO DEFASADO (proposto: 30 membros)

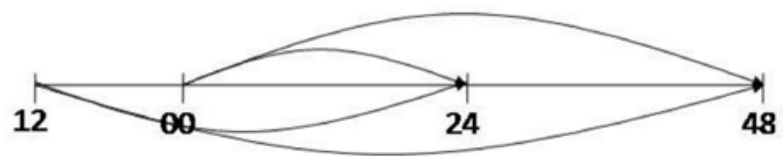

Figura 1 - Descrição esquemática da técnica de previsões defasadas da previsão de tempo por conjunto. 
experimentos são avaliados, sendo três incluindo previsões defasadas, um considerando o EPS-CPTEC/INPE operacional e dois experimentos baseados em previsões determinísticas, com e sem defasagem. Os experimentos são:

o oper $15 \mathrm{~m}$, que corresponde ao conjunto operacional do CPTEC/INPE, com 15 membros inicializados a partir da análise mais recente. Este experimento será considerado como controle para os demais;

o lag $15 \mathrm{~m}$, cujo conjunto defasado é constituído de 15 membros, obtidos a partir das previsões inicializadas $12 \mathrm{~h}$ antes da previsão mais recente;

o lag $30 \mathrm{~m}$, ou conjunto defasado com 30 membros, composto de 15 membros do EPS-CPTEC/INPE, inicializados a partir da análise mais recente e 15 membros, iniciados a partir da análise defasada em 12 horas;

o lag $30 \mathrm{mw}$, similar ao experimento anterior, exceto pelo fato que os membros do conjunto são ponderados pelo inverso do erro quadrático médio na área, média sobre os erros das previsões dos 15 dias anteriores ao da rodada considerada;

o lagdet e o determ, constituídos de previsões determinísticas do modelo de circulação geral atmosférico (MCGA) do CPTEC/INPE, a partir de análises não perturbadas (apenas uma solução), inicializados a partir da análise defasada em $12 \mathrm{~h}$ e da análise mais recente, respectivamente.

Os vários experimentos propostos servem de parâmetro de comparação entre o desempenho das previsões dos

Tabela 1 - Experimentos utilizando a técnica de previsões defasadas

\begin{tabular}{|c|c|c|c|}
\hline Experimentos & No. de Membros & $\begin{array}{c}\text { Previsóes Nåo } \\
\text { Defasadas }\end{array}$ & $\begin{array}{l}\text { Previsóes } \\
\text { Defasadas }\end{array}$ \\
\hline $\log 30 \mathrm{~m}$ & 30 & $\begin{array}{c}15 \text { membros da rodada } \\
\text { mais recente }\end{array}$ & $\begin{array}{c}15 \text { membros } \\
\text { inicializados } 12 \text { horas } \\
\text { antes da rodada mais } \\
\text { recente }\end{array}$ \\
\hline $\log 30 \mathrm{mw}$ & $\begin{array}{c}30 \\
\text { Ponderados pelo } \\
\text { inverso do erro } \\
\text { quadrático médio da } \\
\text { área }\end{array}$ & $\begin{array}{c}15 \text { membros da rodada } \\
\text { mais recente }\end{array}$ & $\begin{array}{c}15 \text { membros } \\
\text { inicializados } 12 \text { horas } \\
\text { antes da rodada mais } \\
\text { recente }\end{array}$ \\
\hline $\operatorname{lag} 15 \mathrm{~m}$ & 15 & - & $\begin{array}{c}15 \text { membros } \\
\text { inicializados } 12 \text { horas } \\
\text { antes da rodada mais } \\
\text { recente }\end{array}$ \\
\hline oper $15 \mathrm{~m}$ & 15 & $\begin{array}{c}15 \text { membros da rodada } \\
\text { mais recente }\end{array}$ & - \\
\hline determ & 1 & $\begin{array}{c}1 \text { membro (análise nåo } \\
\text { perturbada) }\end{array}$ & - \\
\hline lagdet & 1 & - & $\begin{array}{l}1 \text { membro (análise nâo } \\
\text { perturbada de } 12 \text { horas } \\
\text { antes da rodada mais } \\
\text { recente) }\end{array}$ \\
\hline
\end{tabular}


modelos numéricos e estão esquematizados na Tabela 1. Nos experimentos lag $30 \mathrm{~m}$ e lag $30 \mathrm{mw}$, membros defasados são adicionados ao conjunto operacional do EPS-CPTEC/INPE. A realização destes dois experimentos visa avaliar qual é a melhor forma de utilizar as previsões defasadas, ou seja, se há ou não a necessidade de ponderar as previsões mais recentes e defasadas pelos respectivos erros. O experimento oper $15 \mathrm{~m}$, que representa a configuração operacional, é utilizado como referência para comparação com os experimentos lag $30 \mathrm{~m}$ e lag30mw. Será avaliado se o experimento lag30mw indica melhorias significativas sobre o lag $30 \mathrm{~m}$, o que justificaria a sua implementação operacional como fonte adicional de informação ao oper $15 \mathrm{~m}$. Já o experimento lag $15 \mathrm{~m}$ é avaliado para verificar se, em média, as previsões por conjunto defasadas são muito diferentes das previsões dos 15 membros mais recentes (experimento oper15m). Comparação entre os experimentos determ e lagdet também é realizada com a finalidade de verificar se as previsões inicializadas, com 12 horas de diferença, possuem desempenhos significativamente diferentes. Isto ajudará a determinar a necessidade ou não de uma ponderação das previsões ao serem consideradas em um mesmo conjunto.

\subsection{Metodologias de Avaliação}

A avaliação dos resultados deste estudo é baseada essencialmente em índices estatísticos. Os campos utilizados na realização deste estudo são a altura geopotencial em 500 $\mathrm{hPa}$ (Z500), a temperatura do ar no nível de $850 \mathrm{hPa}$ (T850) e a pressão ao nível médio do mar (PNMM). Os índices estatísticos são calculados para as regiões dos extratrópicos e trópicos, definidos como Hemisfério Sul (HS $-90^{\circ} \mathrm{S}$ a $20^{\circ} \mathrm{S}$ ), Hemisfério Norte $\left(\mathrm{HN}-90^{\circ} \mathrm{N}\right.$ a $\left.20^{\circ} \mathrm{N}\right)$ e trópicos (TR $-20^{\circ} \mathrm{N}$ a $20^{\circ} \mathrm{S}$ ), respectivamente. $\mathrm{O}$ enfoque principal deste estudo são os resultados dos extratrópicos do Hemisfério Sul.

Os índices estatísticos utilizados para a avaliação são: a correlação de anomalias $(C A)$, a raiz do erro quadrático médio $(R M S)$, o espalhamento do conjunto ( $S P D)$ e o coeficiente de correlação linear $(r)$, entre a $C A$ e o $S P D$. Estes índices são brevemente descritos a seguir.

\section{a) Conjunto Médio}

A fim de comparar o desempenho das previsões do conjunto e a previsão de controle foi calculado o conjunto médio para cada ponto de grade $j$ do modelo, de acordo com a equação 1):

$$
E M_{j}=\frac{1}{N} \sum_{i=1}^{N} F_{j}^{i}
$$

onde $i$ é o índice para a previsão de cada membro, $N$ o número total de membros do conjunto, $F_{j}^{i}$ o campo previsto por cada membro $i$ para cada ponto $j$ e $E M_{j}$ a previsão do conjunto médio.

\section{b) Correlação de Anomalias}

A $C A$ é uma medida freqüentemente usada para avaliar o desempenho de modelos de previsão, e é definida como a correlação entre uma anomalia (isto é, diferença em relação à climatologia) de um campo previsto e uma anomalia do campo da análise de verificação, ou seja, a correlação de anomalias é a razão entre a covariância e a raiz quadrada do produto de duas variâncias esperadas:

$$
C A=100 \times \frac{\sum_{j=1}^{J}\left[\left(F_{j}-C_{j}\right)^{\prime} \times\left(A_{j}-C_{j}\right)^{\prime}\right] \times W_{j}}{\left\{\sum_{j=1}^{J}\left[\left(F_{j}-C_{j}\right)^{2} \times W_{j}\right] \times \sum_{j=1}^{J}\left[\left(A_{j}-C_{j}\right)^{2} \times W_{j}\right]\right\}^{\frac{1}{2}}}
$$

onde: ( )' significa o desvio da média na área, $j$ é o índice para cada ponto de grade sobre as regiões de avaliação neste estudo (HN, TR e HS), $J$ o número total de pontos na área de estudo, $W_{j}$ o peso para a área que o ponto representa, definido como sendo o cosseno da latitude de cada ponto de grade $j . F_{j}$ é o campo previsto, $A_{j}$ o campo analisado, $C_{j}$ o campo climatológico, $F$ ${ }_{j}-C_{j}$ é a anomalia prevista para o ponto $j$ e $A_{j}-C_{j}$ a anomalia observada no ponto $j$.

\section{c) Espalhamento}

O SPD fornece uma estimativa da confiabilidade ou incerteza de uma previsão. É definido como a distância média quadrática dos membros do conjunto em relação ao conjunto médio e é calculado conforme a equação:

$$
C A=100 \times \frac{\sum_{j=1}^{J}\left[\left(F_{j}-C_{j}\right) \times\left(A_{j}-C_{j}\right)\right] \times W_{j}}{\left\{\sum_{j=1}^{J}\left[\left(F_{j}-C_{j}\right)^{2} \times W_{j}\right] \times \sum_{j=1}^{J}\left[\left(A_{j}-C_{j}\right)^{2} \times W_{j}\right]\right\}^{\frac{1}{2}}}
$$

onde os campos e os índices da equação (3) assumem o significado descrito anteriormente.

\section{d) Raiz do Erro Quadrático Médio}

A $R M S$ é definida como sendo a raiz quadrada da razão entre a soma das diferenças quadráticas entre os campos previstos $F_{j}$ e analisados $A_{j}$, ponderado pelo peso $W_{j}$ de cada ponto de grade, e a soma dos pesos $W_{j}$, da área avaliada. 


$$
R M S=\left\{\frac{\sum_{j=1}^{J}\left(F_{j}-A_{j}\right)^{2} \times W_{j}}{\sum_{j=1}^{J} W_{j}}\right\}^{1 / 2}
$$

\section{e) Relação entre o Desempenho do EPS-CPTEC/ INPE e o Espalhamento}

O $r$ entre a correlação de anomalias e o espalhamento do conjunto é calculado através da expresão:

$$
R M S=\left\{\frac{\sum_{j=1}^{J}\left(F_{j}-A_{j}\right)^{2} \times W_{j}}{\sum_{j=1}^{J} W_{j}}\right\}^{1 / 2}
$$

onde $S P D$ e $C A$ são o espalhamento e o desempenho da previsão, respectivamente, e $K$ é o número de pares das variáveis $S P D$ e $C A$. O circunflexo sobre as variáveis indica média no período considerado, ou seja, sobre o número de pares $K$ de $S P D$ e $C A$.

O cálculo de r é utilizado para avaliar as relações baixo espalhamento/alto desempenho e alto espalhamento/baixo desempenho ou, em outras palavras, se quando $S P D$ foi maior do que SPD, o desempenho do conjunto $(C A)$ foi menor do que $C \hat{A}$ e vice-versa. A referência para indicar alto ou baixo espalhamento/ desempenho é a média do espalhamento e do desempenho do período considerado. O coeficiente de correlação pode variar de -1 a 1 , entretanto, são os valores negativos do coeficiente de correlação entre $S P D$ e $C A$ que mostram que o espalhamento pode ser utilizado como um indicador da confiabilidade da previsão.

\section{RESULTADOS}

Nesta seção, os resultados dos experimentos realizados são apresentados e discutidos. Nas subseções 3.1 e 3.2 os desempenhos dos experimentos são avaliados e comparados entre si, em termos da correlação de anomalias e da raiz do erro quadrático médio, respectivamente, e na subseção 3.3 é discutida a relação entre o desempenho do conjunto médio e o espalhamento do conjunto.

\subsection{Desempenho dos Experimentos Usando Correlação de Anomalias}

Nas Figuras 2, 3 e 4 são apresentadas as correlações de anomalias dos campos de altura geopotencial (Z500), temperatura do ar em $850 \mathrm{hPa}$ (T850) e pressão ao nível médio do mar (PNMM), respectivamente, para cada prazo de previsão e para as regiões (a) HN, (b) TR e (c) HS.

Observa-se que nos extratrópicos (HN e HS), os desempenhos dos experimentos com previsões por conjunto (oper15m, lag15m, lag30m e lag30mw) são melhores do que os desempenhos dos experimentos com previsões determinísticas (determ e lagdet), para os três campos analisados e para todos os prazos de previsão.

Os desempenhos dos experimentos em que as previsões por conjunto mais recentes são combinadas com as previsões
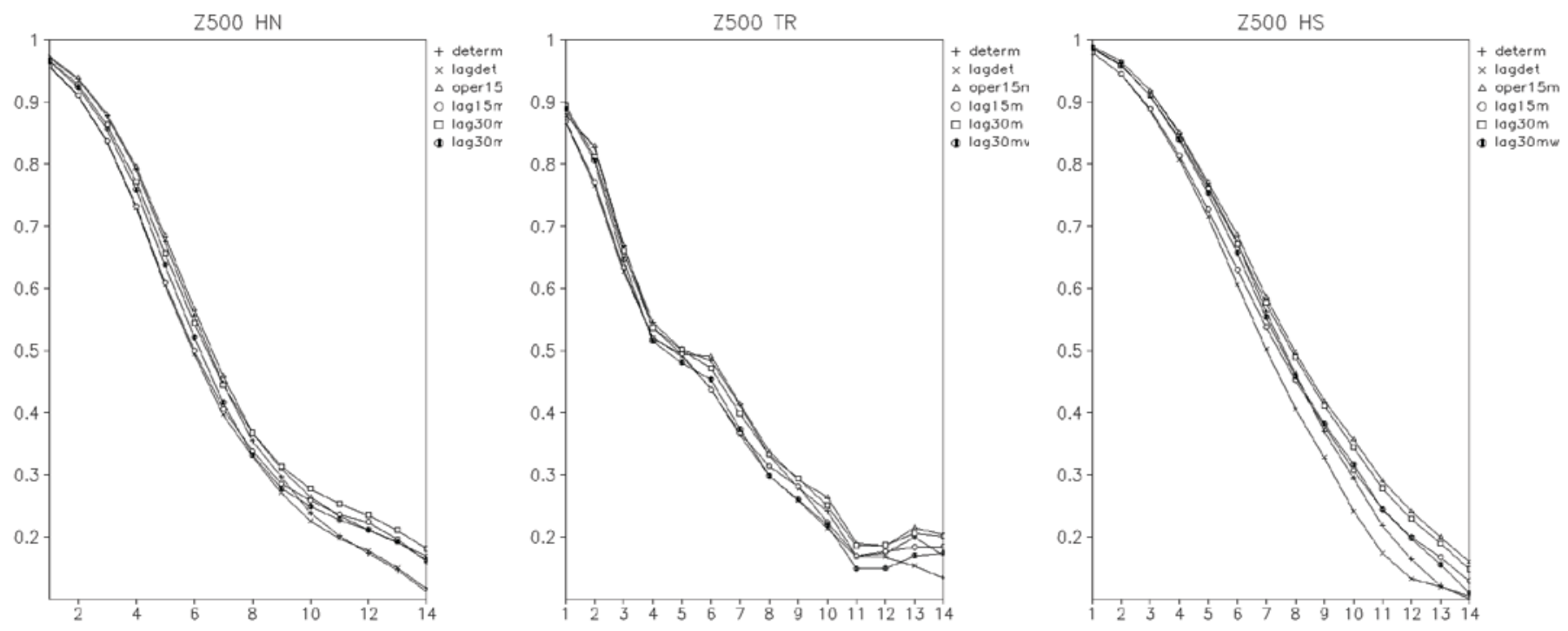

Figura 2 - Correlação de anomalias da altura geopotencial em $500 \mathrm{hPa}$ (ordenada - y) em função do prazo de previsão (abscissa - x) para os vários experimentos: (a) HN, (b) TR e (c) HS. A unidade do eixo x é dias. 
por conjunto defasadas em 12h, sem (lag30m) e com (lag30mw) ponderação pelos erros, mostram que o experimento lag $30 \mathrm{~m}$ apresenta resultados melhores ou semelhantes ao do lag30mw, tanto no HN quanto no HS, para os três campos considerados (Figuras 2a,c; 3a,c; 4a,c). Esta aparente discrepância entre os resultados pode estar associada à forma como o peso de cada conjunto de previsões está sendo calculado. Neste trabalho, os pesos foram obtidos como sendo a média do $R M S$ das 15 previsões mais recentes, mediado na área considerada. Isto implica em assumir que o erro é uniforme para todos os pontos de grade de cada região. Talvez se os pesos fossem calculados para cada ponto de grade, os resultados seriam diferentes. Esta investigação será realizada posteriormente em outro estudo, pois o objetivo deste trabalho é mostrar que a utilização simples das previsões defasadas em $12 \mathrm{~h}$ pode ser utilizada para adicionar informações úteis às previsões por conjunto operacionais do CPTEC/INPE, o que é verificado nos resultados apresentados nas Figuras 2, 3 e 4.
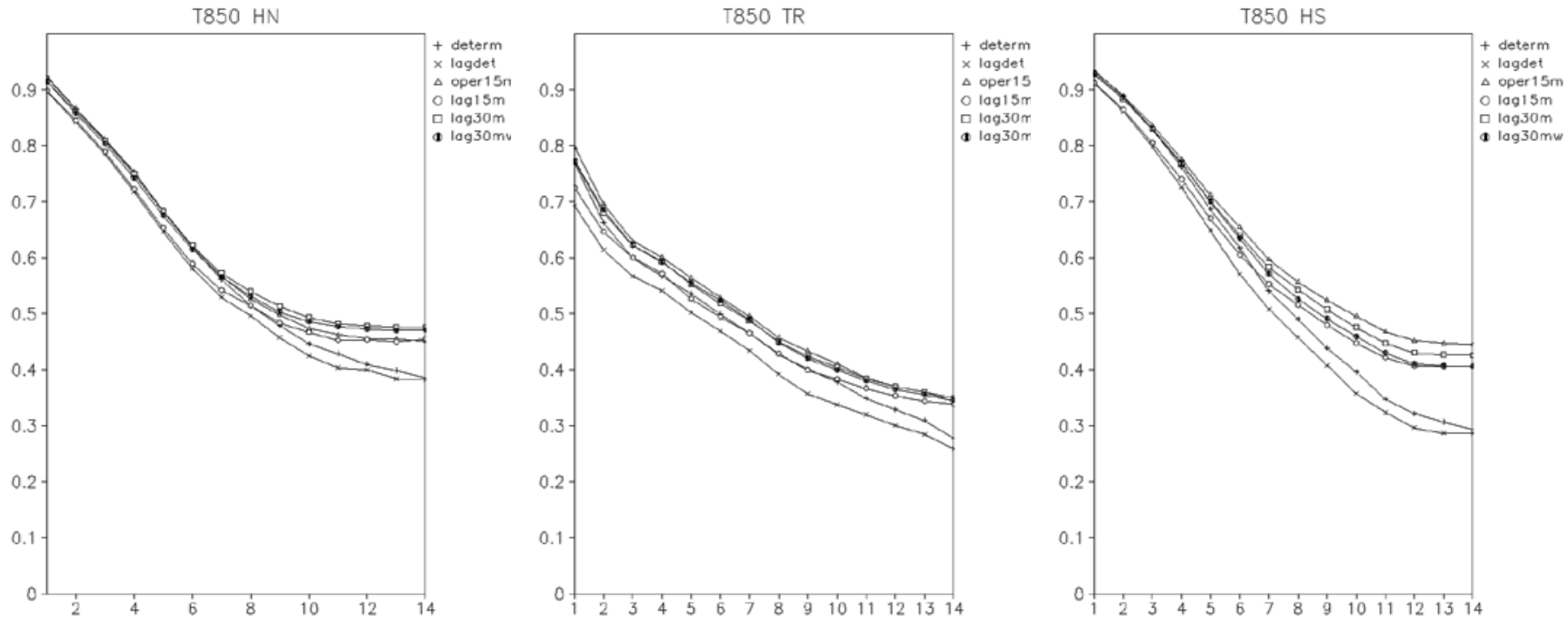

Figura 3 - Correlação de anomalias da temperatura do ar em $850 \mathrm{hPa}$ (ordenada - y) em função do prazo de previsão (abscissa - x) para os vários experimentos: (a) HN, (b) TR e (c) HS. A unidade do eixo x é dias.
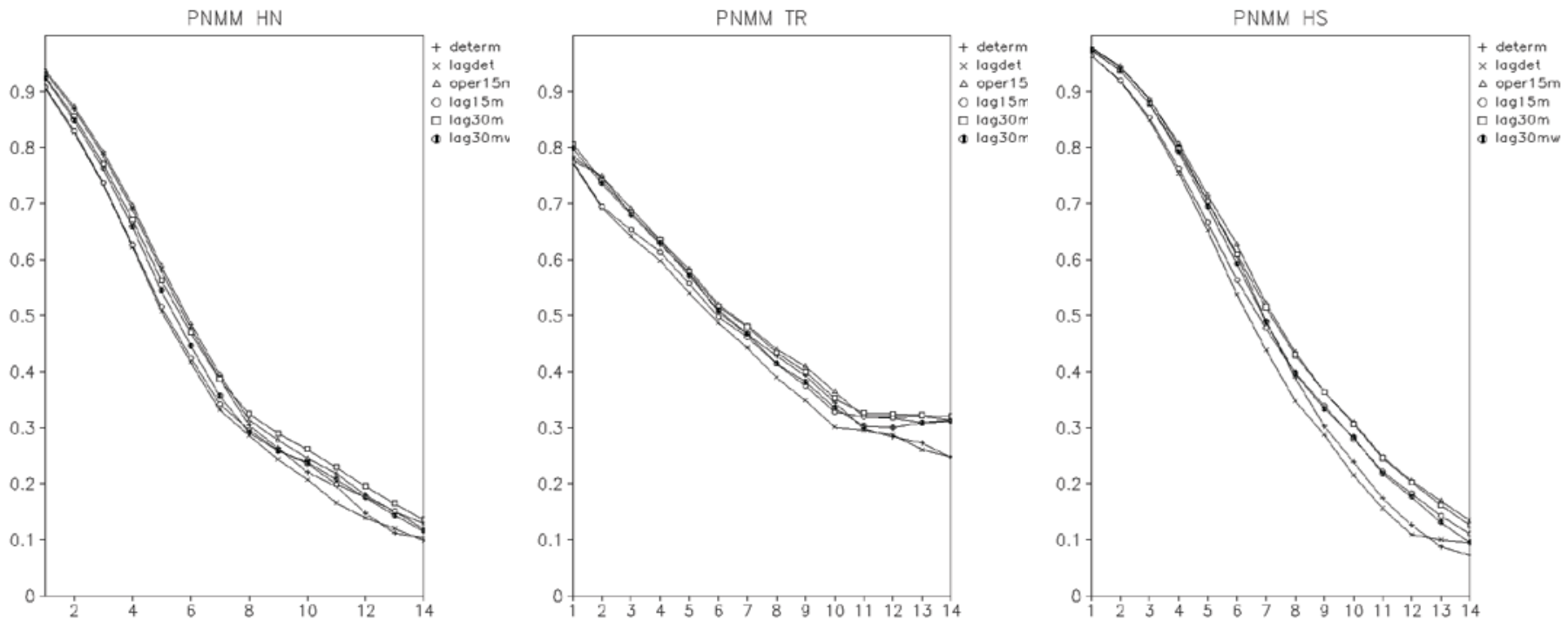

Figura 4 - Correlação de anomalias da pressão ao nível médio do mar (ordenada - y) em função do prazo de previsão (abscissa - x) para os vários experimentos: (a) HN, (b) TR e (c) HS. A unidade do eixo x é dias. 


\subsection{Comparação dos Desempenhos dos Experimentos através da Raiz do Erro Quadrático Médio e do Espalhamento}

O $R M S$ e o $S P D$ das previsões de cada um dos experimentos, para as variáveis Z500, T850 e PNMM são apresentados nas Figuras 5, 6 e 7, respectivamente.

Uma análise geral dos valores de $R M S$ de cada experimento indica que os resultados estão de acordo com àqueles obtidos em termos de $C A$, ou seja, menores erros são verificados nos experimentos que utilizam previsões por conjunto. É importante observar que os resultados são similares para todas as variáveis analisadas. Destaca-se o desempenho ligeiramente superior do experimento lag $30 \mathrm{~m}$ em relação ao experimento oper $15 \mathrm{~m}$, tanto em termos de $R M S$ (quanto menor melhor), quanto em termos de $S P D$ que, de maneira geral, quanto mais próximo for do valor do $R M S$, melhor é o seu desempenho (Figuras. 5a,c; 6a,c e 7a,c). O resultado mais relevante é, certamente, o impacto da utilização de previsões defasadas no $S P D$ das previsões, principalmente nos extratrópicos do $\mathrm{HN}$
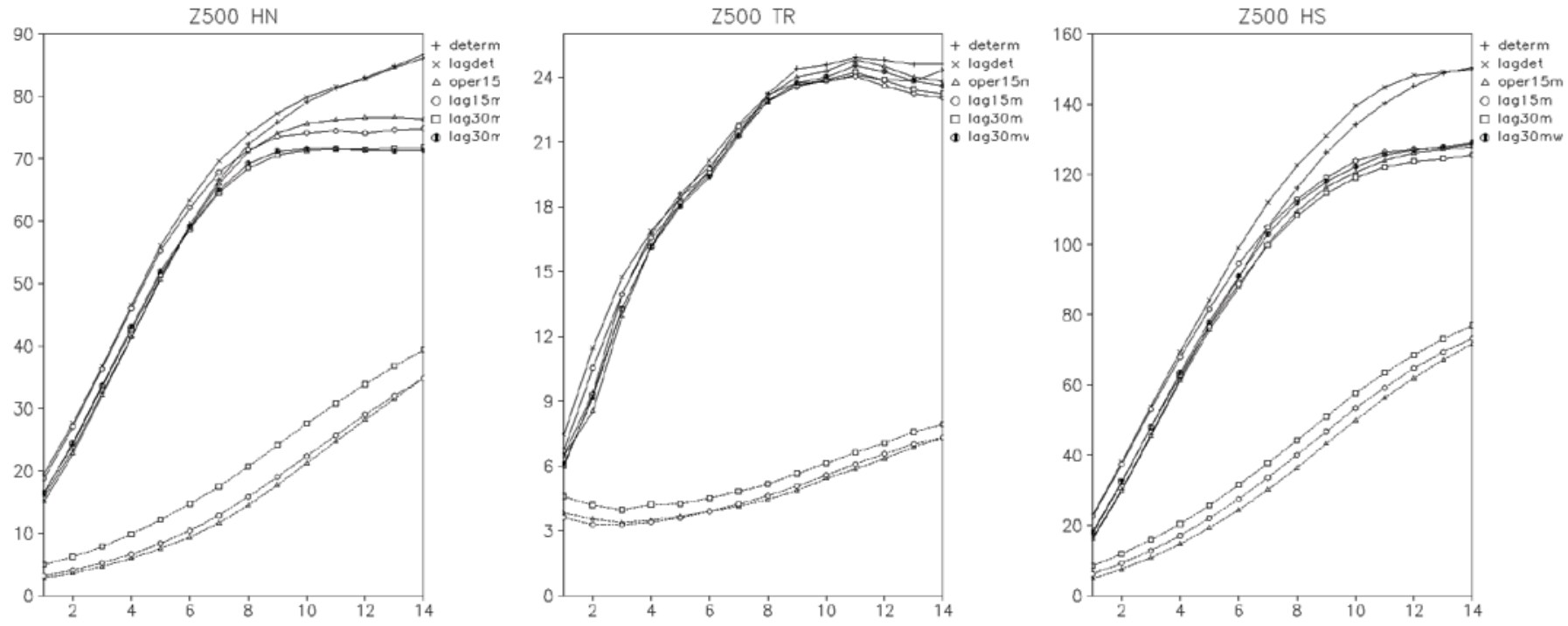

Figura 5 - Raiz do erro quadrático médio (linhas contínuas) e espalhamento (linhas tracejadas) do campo de altura geopotencial em 500 hPa (ordenada - y), em função do prazo de previsão (abscissa - x), para os diferentes experimentos: (a) HN, (b) TR e (c) HS. As unidades dos eixos y e x são m e dias, respectivamente.
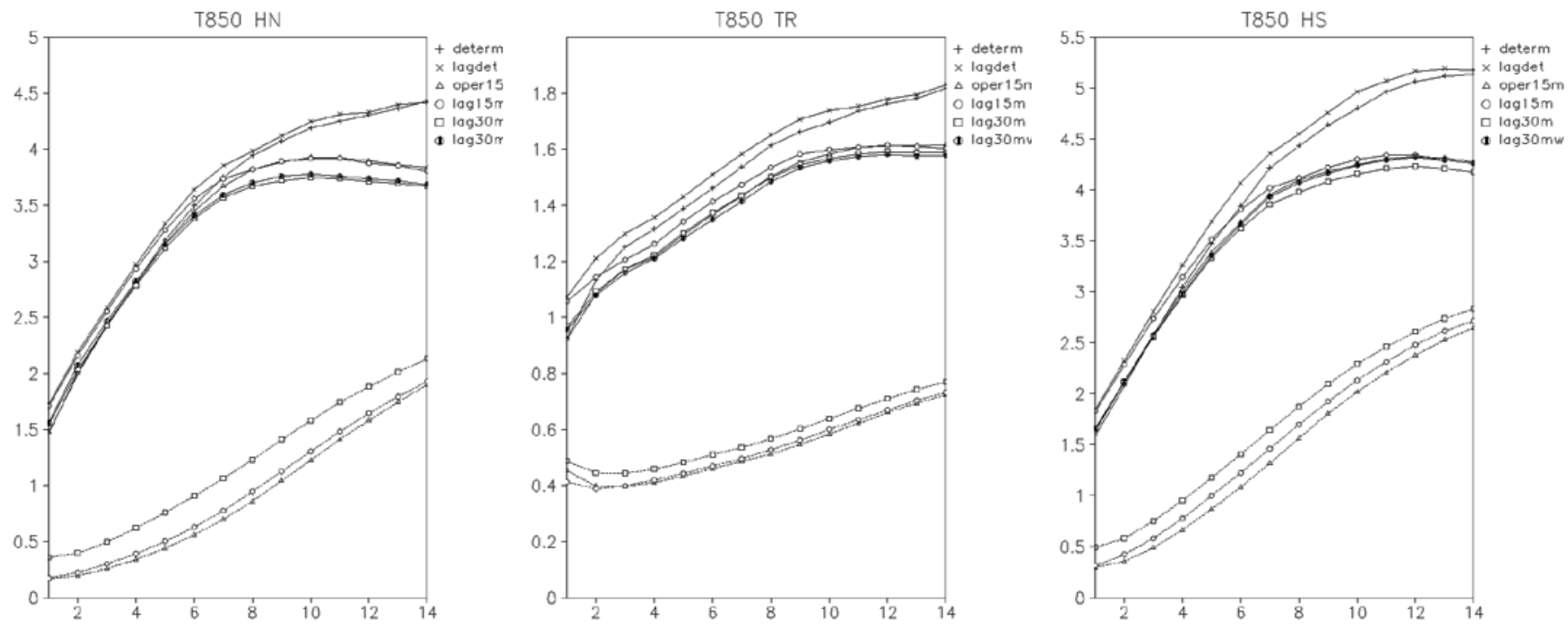

Figura 6 - Raiz do erro quadrático médio (linhas contínuas) e espalhamento (linhas tracejadas) do campo de temperatura do ar em $850 \mathrm{hPa}$ (ordenada - y), em função do prazo de previsão (abscissa - x), para os diferentes experimentos: (a) HN, (b) TR e (c) HS. As unidades dos eixos y e x são K e dias, respectivamente. 
e do HS, como pode ser observado nas Figuras 5a,c; 6a,c e 7a,c para as variáveis Z500, T850 e PNMM, respectivamente. Nota-se que no experimento lag30m, o espalhamento das previsões é aumentado em praticamente todos os prazos de previsão. $\mathrm{O}$ aumento do espalhamento, juntamente com uma ligeira diminuição do $R M S$ das previsões, indica que a inclusão das previsões defasadas contribui para tornar as previsões por conjunto do CPTEC/INPE mais balanceadas, ou seja, ajudam a diminuir a subdispersão do sistema, o que provavelmente refletirá em previsões de probabilidades melhores.

\subsection{Relação entre o Espalhamento e o Desempenho do Conjunto}

A relação entre o $S P D$ e a $C A$ é avaliada a partir das seguintes análises: i) o diagrama de dispersão do $S P D$ versus $C A$, calculando a correlação linear $r$ entre estas variáveis; e ii) a tabela de contingência, baseada no número de ocorrências que se encontram nos diferentes quadrantes selecionados a partir das medianas.

Os diagramas de dispersão do espalhamento/desempenho do conjunto do HS são apresentados nas Figuras 8, 9 e 10, para as variáveis Z500, T850 e PNMM, respectivamente. Embora a avaliação tenha sido realizada para todos os prazos de previsão, neste estudo são apresentados os resultados para os prazos de previsão de 5 e 7 dias, para os experimentos (a) oper $15 \mathrm{~m}$ e (b) lag30m. O diagrama de dispersão é dividido pelos valores medianos do espalhamento e do desempenho do conjunto, linhas horizontal e vertical que dividem os gráficos em quatro quadrantes. Os quadrantes são numerados no sentido horário, começando no canto superior esquerdo. $\mathrm{O}$ número de pontos contidos em cada quadrante é apresentado nos vértices da figura. Isto permite construir uma tabela de contingência $2 \times 2$ para os valores de espalhamento grande/pequeno e de desempenho alto/baixo (Tabela 2). Nota-se que, usando a mediana para definir as categorias da tabela de contingência, obtém-se uma tabela necessariamente simétrica (Molteni et al, 1996). As áreas 1 e 3 representam o resultado desejado, isto é, quando há correspondência inversa entre o espalhamento e o desempenho da previsão: os pontos da área 1 indicam o número de vezes em que o espalhamento foi pequeno e o desempenho foi alto (XmeYma), enquanto que, os pontos da área 3 representam o número de vezes em que o espalhamento foi grande e o desempenho foi baixo (XmaYme). As áreas 2 e 4 representam os erros, isto é, os casos em que esta correspondência inversa não foi verificada.

Analisando as Figuras 8, 9 e 10, observa-se que a diagonal entre os quadrantes 1 e 3 é mais populosa do que a dos quadrantes 2 e 4 , para os prazos de previsão mostrados. Isto indica que há uma relação inversa entre o espalhamento e o desempenho do conjunto, ou seja, na maioria dos casos, o pequeno (grande) espalhamento dos membros do conjunto está associado com alto (baixo) desempenho do conjunto médio.

As Tabelas 2, 3 e 4 mostram a freqüência absoluta, a freqüência relativa, entre parêntesis, e o coeficiente de correlação ( $r$ ) entre o $S P D$ e a $C A$ dos experimentos lag $30 \mathrm{~m}$ e oper15m, para os prazos de previsão de 5, 7, 9 e 13 dias, nos extratrópicos do HS. Nas tabelas são apresentadas as quatro
PNMM HN

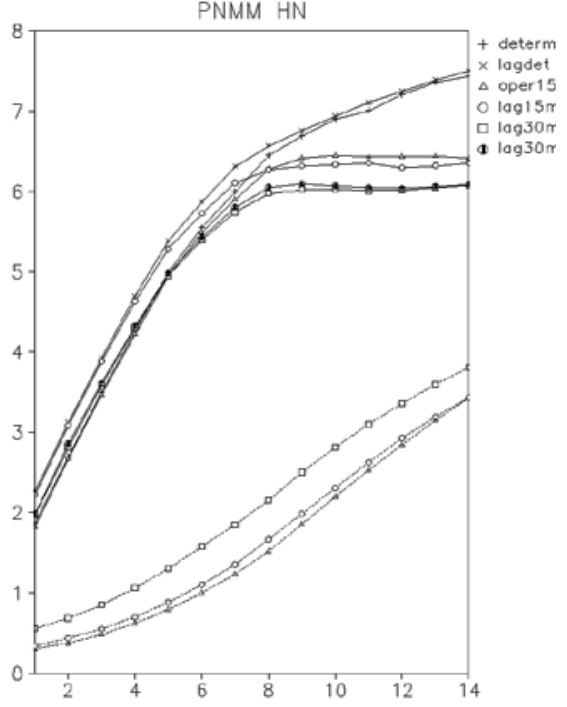

PNMM TR

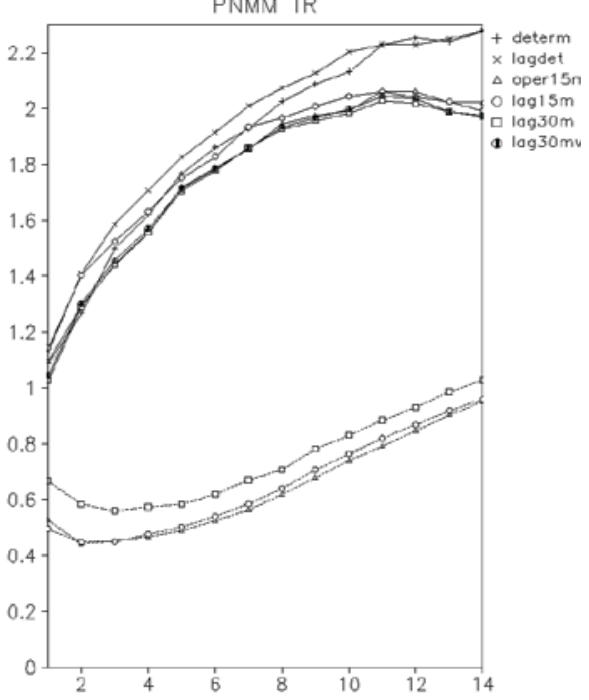

PNMM HS

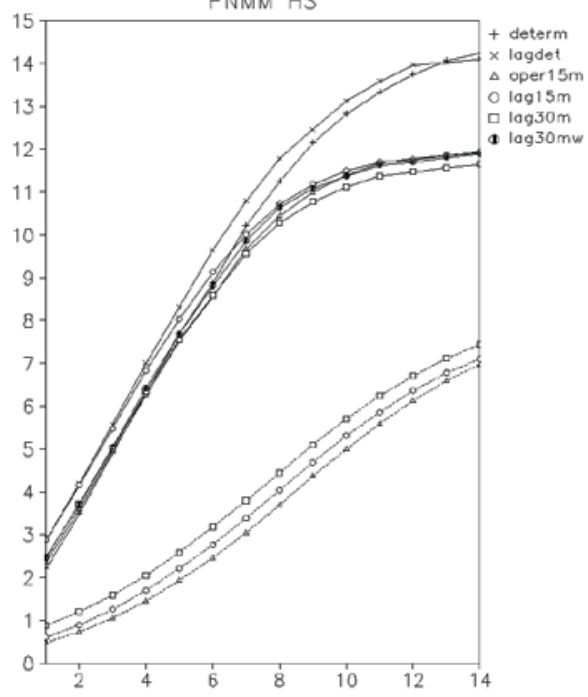

Figura 7 - Raiz do erro quadrático médio (linhas contínuas) e espalhamento (linhas tracejadas) do campo de pressão ao nível médio do mar (ordenada - y), em função do prazo de previsão (abscissa - x), para os diferentes experimentos: (a) HN, (b) TR e (c) HS. As unidades dos eixos y e x são hPa e dias, respectivamente. 
combinações dos valores de $\mathrm{x}(S P D)$ e y $(C A)$, maiores (Xma, Yma) ou menores (Xme, Yme) que a mediana.

Comparando os vários prazos de previsão das Tabelas 2, 3 e 4 de contingência, observa-se que o prazo de 7 dias apresenta a maior freqüência de casos de $C A$ alta e $S P D$ pequeno, com valores de $27 \%$ e $32 \%$ na variável $Z 500,32 \%$ e $34 \%$ na variável T850 e $30 \%$ e $29 \%$ na variável PNMM, nos experimentos
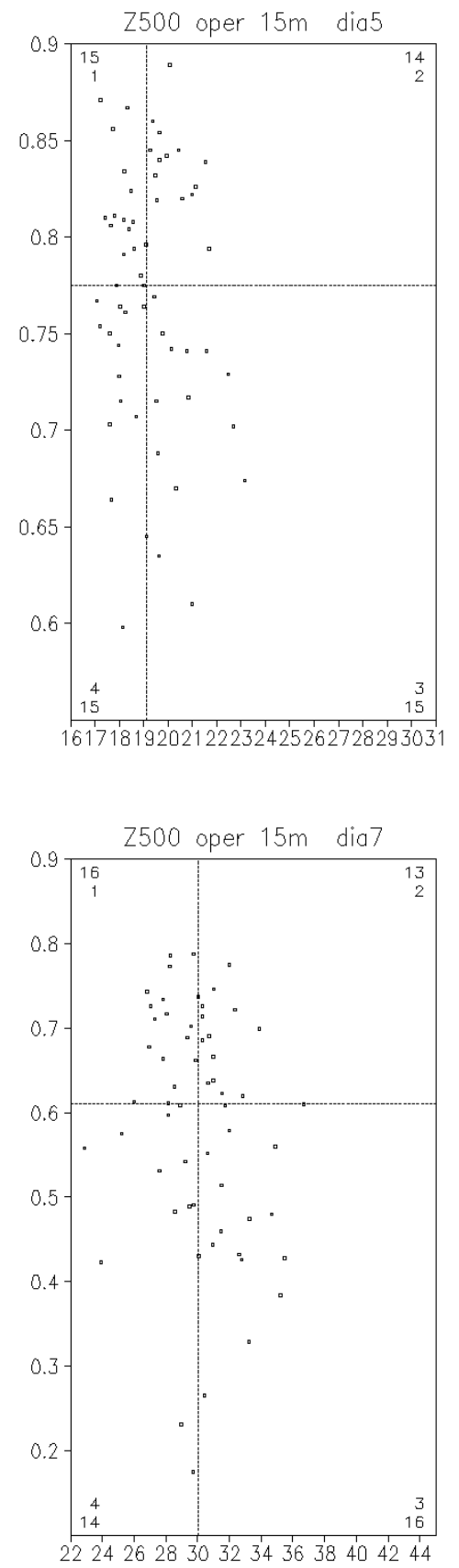

oper $15 \mathrm{~m}$ e lag $30 \mathrm{~m}$, respectivamente. Este prazo também apresentou a maior correlação inversa entre estas variáveis, com valores de $r$ de $-0,20$ para Z500, $-0,30$ para T850, e $-0,40$ para PNMM para ambos os experimentos.

Os valores de $r$, embora baixos, estão de acordo com os encontrados na literatura. Mendonça e Bonatti (2004) encontraram valores de $r$ de $-0,086$ e $-0,067$ para o quinto e
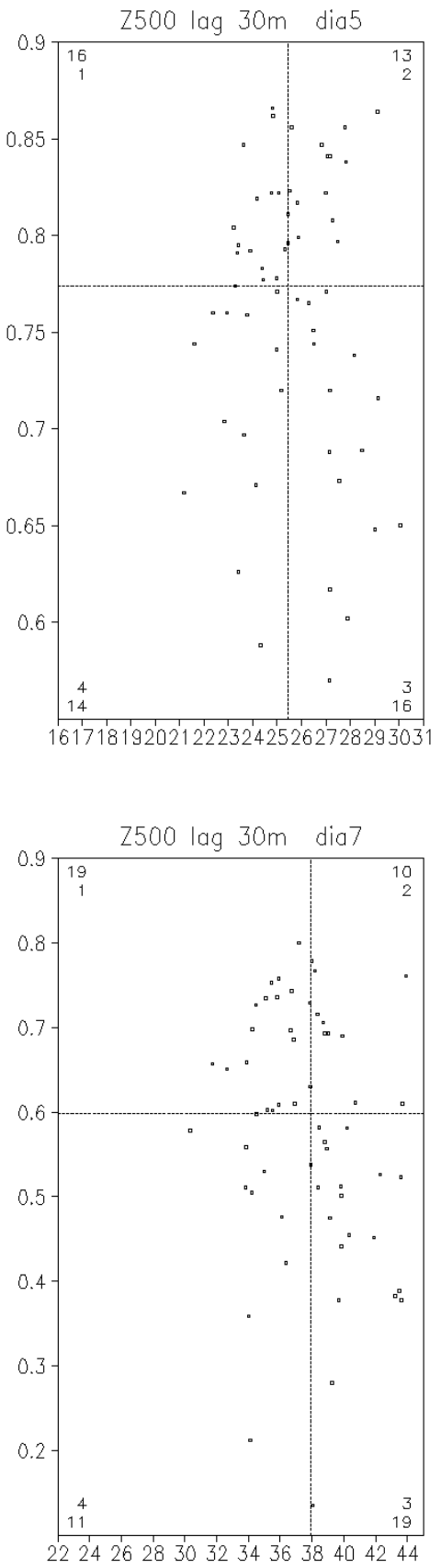

Figura 8 - Diagrama de dispersão entre o desempenho (ordenada) versus espalhamento da previsão (abscissa) da altura geopotencial em $500 \mathrm{hPa}$ (Z500) no HS, para $5^{\circ}$ e $7^{\circ}$ dia de previsão dos experimentos: oper $15 \mathrm{~m}$ (a) e (c) e lag30m (b) e (d), respectivamente, em uma amostra de 59 dias válidos. 
sétimo dia de previsão, respectivamente, usando previsões por conjunto na resolução de T062L28 do modelo do CPTEC/INPE, para o período de outubro de 2001 a setembro de 2003. Molteni e Palmer (1991) obtiveram valores de correlação linear entre 0,3 e 0,4 para o prazo de previsão de 7 dias, no Hemisfério Norte. Molteni et al. (1996) encontraram valores de correlação de 0,56 e 0,59, para o inverno e verão, respectivamente, para o EPS do ECMWF, usando valores de $R M S$ médio nos prazos de 5 a 7 dias.

Whitaker e Loughe (1998) encontraram maiores valores de correlação entre o $R M S$ e o SPD para os prazos de 5 e 6 dias. No trabalho de Barker (1991), maiores valores de correlação foram obtidos em torno do prazo de previsão de 12 dias. Segundo estes trabalhos, num sistema de previsão por conjunto perfeito,
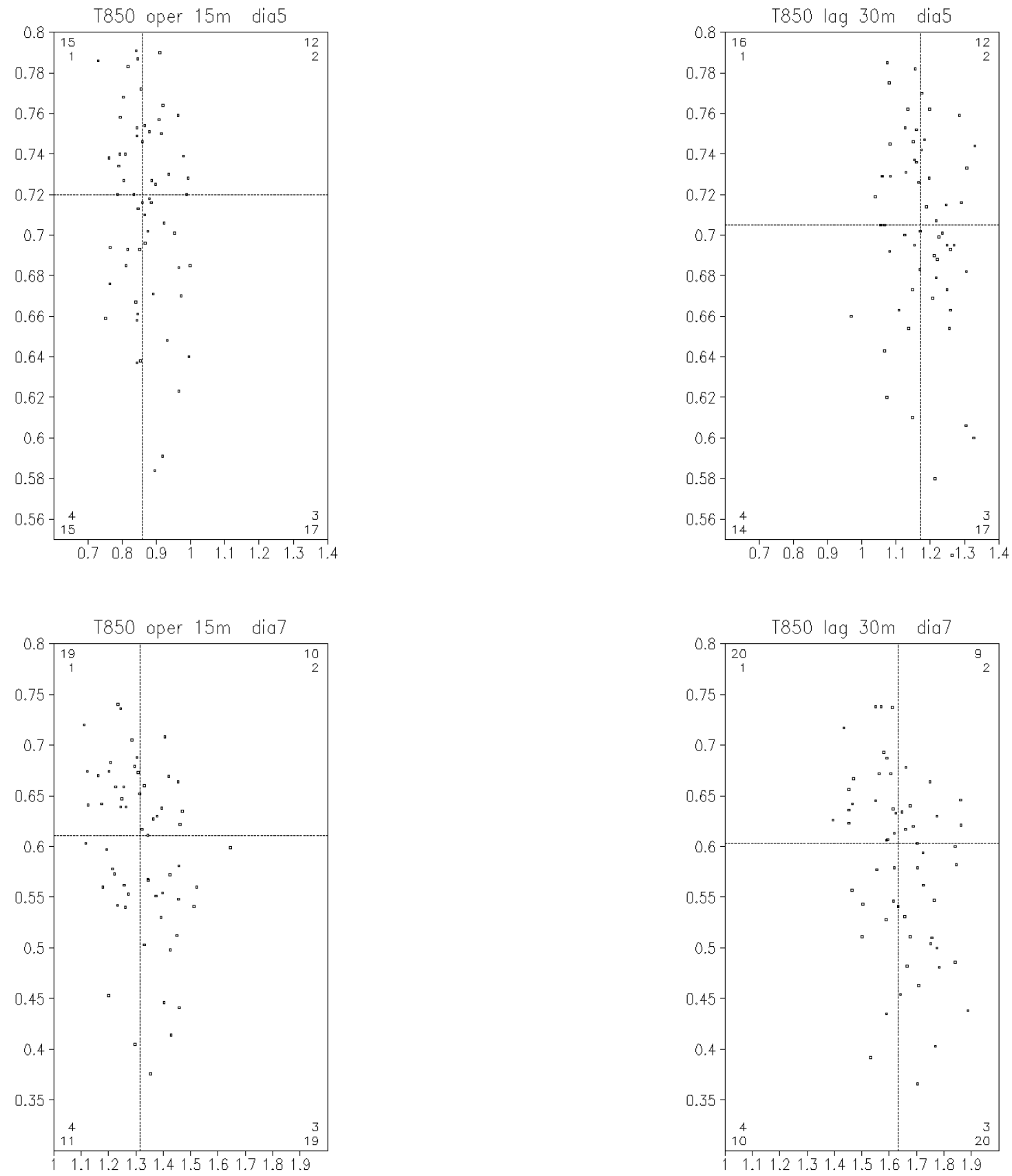

Figura 9 - Diagrama de dispersão entre o desempenho (ordenada) versus espalhamento da previsão (abscissa) da temperatura no nível de 850 hPa (T850) no HS, para $5^{\circ}$ e $7^{\circ}$ dia de previsão dos experimentos: oper15m (a) e (c) e lag30m (b) e (d), respectivamente, em uma amostra de 59 dias válidos. 
a variabilidade diária do espalhamento e então, a correlação erro/espalhamento pode se originar de uma das duas fontes: 1) variação diária na amplitude do erro da análise; 2) variação diária na taxa de crescimento da perturbação inicial associada com a variação na instabilidade atmosférica. Para previsões de tempo de curto prazo (1 a 3 dias) a fonte 1 é dominante, enquanto que, a 2 domina para prazos longos. Como a variação das amplitudes das perturbações não está bem correlacionada com as variações diárias dos erros das previsões de curto prazo, é provável que as perturbações iniciais não estejam representando adequadamente as variações diárias do erro da análise. Desta forma, as correlações $R M S / S P D$ estão associadas às variações diárias na instabilidade atmosférica. Como este mecanismo não pode produzir variabilidade no espalhamento para prazos curtos, e para prazos longos a correlação $R M S / S P D$ deve se aproximar de zero, a correlação entre o $R M S / S P D$ deve ter um
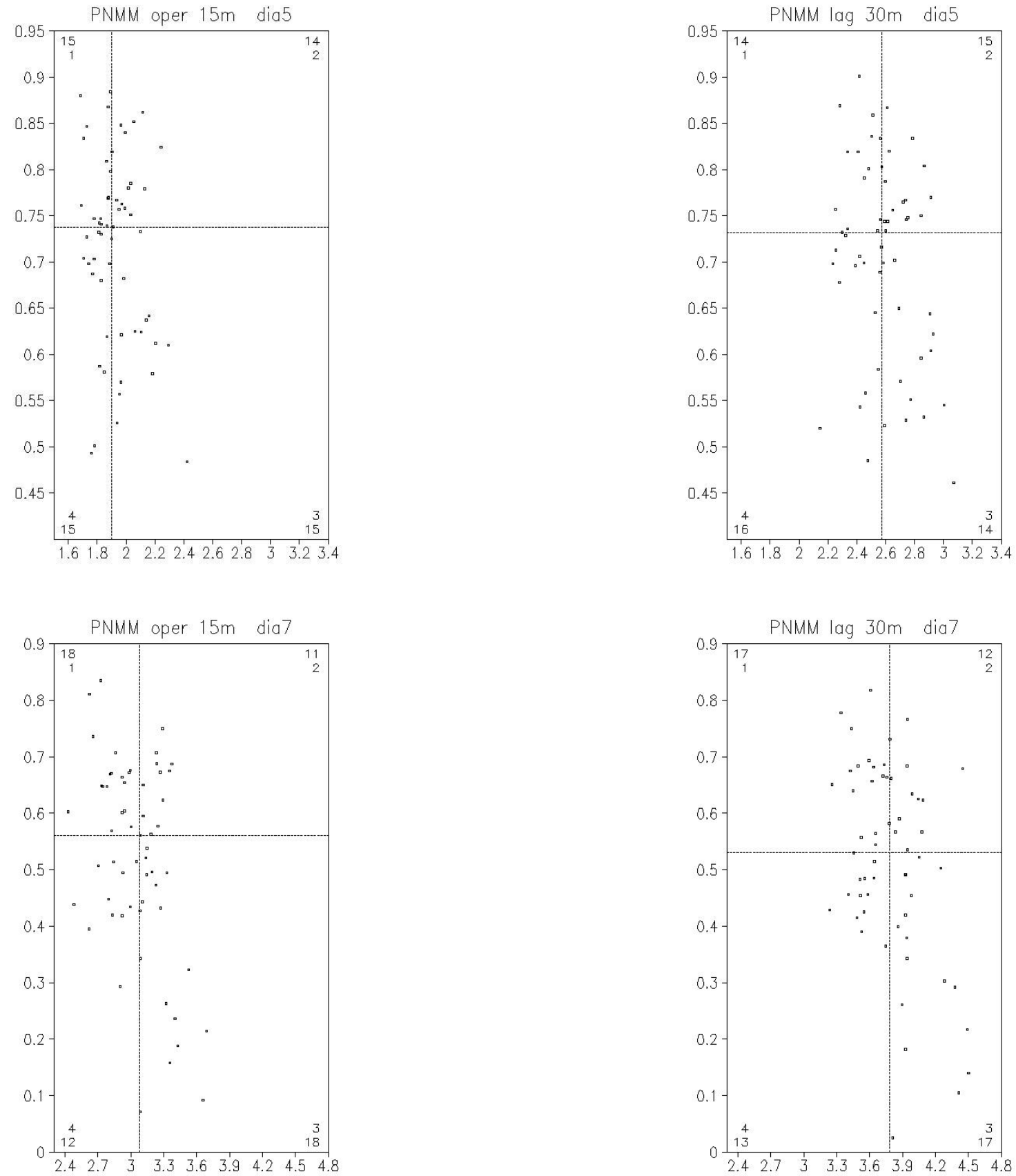

Figura 10 - Diagrama de dispersão entre o desempenho (ordenada) versus espalhamento da previsão (abscissa) da pressão ao nível do mar (PNMM) no HS, para 5o e 7 o dia de previsão dos experimentos: oper $15 \mathrm{~m}$ (a) e (c) e lag30m (b) e (d), respectivamente, em uma amostra de 59 dias válidos. 
Tabela 2 - Tabela de contingência para o espalhamento e desempenho da previsão do conjunto operacional (oper $15 \mathrm{~m})$ e defasado (lag30m), para os vários prazos de previsão, no HS. São apresentados os valores absolutos e relativos (entre parêntesis) de cada categoria, baseados na mediana, e o coeficiente de correlação (r) entre o espalhamento e desempenho da previsão. Os valores de espalhamento pequeno e desempenho alto são grifados.

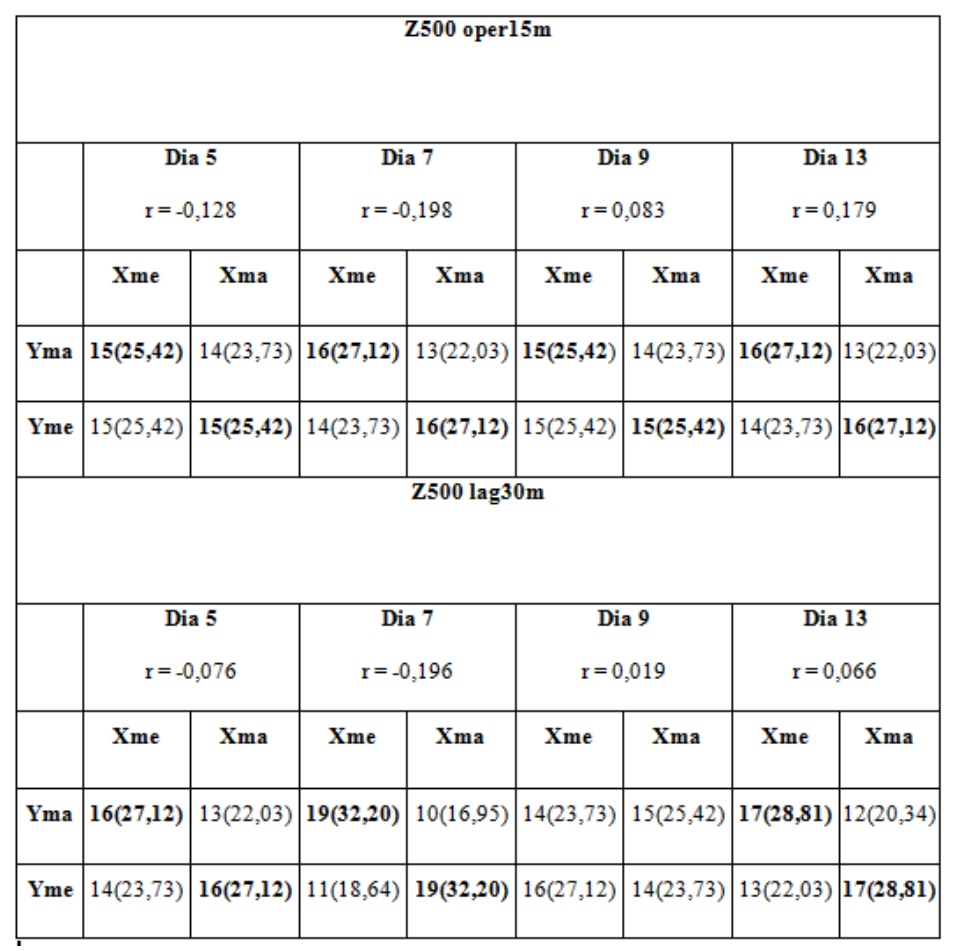

Tabela 3 - Tabela de contingência para o espalhamento e desempenho da previsão da T850 do conjunto operacional (oper $15 \mathrm{~m}$ ) e defasado (lagged30m), para os vários prazos de previsão, no HS. São apresentados os valores absolutos e relativos (entre parêntesis) de cada categoria, baseados na mediana, e o coeficiente de correlação (r) entre o espalhamento e desempenho da previsão. Os valores de espalhamento pequeno e desempenho alto são grifados.

\begin{tabular}{|c|c|c|c|c|c|c|c|c|}
\hline \multicolumn{9}{|c|}{ T850 oper15m } \\
\hline & \multicolumn{2}{|c|}{$\begin{array}{c}\text { Dia } 5 \\
r=-0,203\end{array}$} & \multicolumn{2}{|c|}{$\begin{array}{c}\text { Dia } 7 \\
r=-0,315\end{array}$} & \multicolumn{2}{|c|}{$\begin{array}{c}\text { Dia } 9 \\
\mathrm{r}=-0,105\end{array}$} & \multicolumn{2}{|c|}{$\begin{array}{c}\text { Dia } 13 \\
r=0,205\end{array}$} \\
\hline & Xme & Xma & Xme & Xma & Xme & Xma & Xme & Xma \\
\hline Yma & $15(25,42)$ & $12(20,34)$ & $19(32,20)$ & $10(16,94)$ & $15(25,42)$ & $14(23,73)$ & $15(25,42)$ & $13(22,03)$ \\
\hline Yme & $15(25,42)$ & $17(28,81)$ & $11(18,64)$ & $19(32,20)$ & $15(25,42)$ & $15(25,42)$ & $15(25,42)$ & $16(27,12)$ \\
\hline \multicolumn{9}{|c|}{ T850 lag30m } \\
\hline & \multicolumn{2}{|c|}{$\begin{array}{c}\text { Dia } 5 \\
\mathrm{r}=-0,213\end{array}$} & \multicolumn{2}{|c|}{$\begin{array}{c}\text { Dia } 7 \\
r=-0,335\end{array}$} & \multicolumn{2}{|c|}{$\begin{array}{c}\text { Dia } 9 \\
r=-0,108\end{array}$} & \multicolumn{2}{|c|}{$\begin{array}{c}\text { Dia 13 } \\
\mathrm{r}=-0,120\end{array}$} \\
\hline & Xme & Xma & Xme & Xma & Xme & Xma & Xme & Xma \\
\hline Yma & $16(27,12)$ & $12(20,34)$ & $20(33,90)$ & $2(15,25)$ & $17(28,81)$ & $12(20,34)$ & $17(28,81)$ & $12(20,34)$ \\
\hline Yme & $14(23,73)$ & $17(28,81)$ & $10(16,95)$ & $20(33,90)$ & $13(22,03)$ & $17(28,81)$ & $13(22,03)$ & $17(28,81)$ \\
\hline
\end{tabular}


Tabela 4 - Tabela de contingência para o espalhamento e desempenho da previsão da PNMM do conjunto operacional (oper15m) e defasado (lagged $30 \mathrm{~m}$ ), para os vários prazos de previsão, no HS. São apresentados os valores absolutos e relativos (entre parêntesis) de cada categoria, baseados na mediana, e o coeficiente de correlação (r) entre o espalhamento e desempenho da previsão. Os valores de espalhamento pequeno e desempenho alto são grifados.

\begin{tabular}{|c|c|c|c|c|c|c|c|c|}
\hline \multicolumn{9}{|c|}{ PNMM oper15m } \\
\hline & \multicolumn{2}{|c|}{$\begin{array}{c}\text { Dia } 5 \\
\mathrm{r}=-0,213\end{array}$} & \multicolumn{2}{|c|}{$\begin{array}{c}\text { Dia } 7 \\
r=-0,425\end{array}$} & \multicolumn{2}{|c|}{$\begin{array}{c}\text { Dia } 9 \\
r=-0,251\end{array}$} & \multicolumn{2}{|c|}{$\begin{array}{c}\text { Dia 13 } \\
\mathrm{r}=-0,236\end{array}$} \\
\hline & Xme & Xma & Xme & Xma & Xme & Xma & Xme & Xma \\
\hline Yma & $15(25,42)$ & $14(23,73)$ & $18(30,31)$ & $11(18,64)$ & $17(28,81)$ & $12(20,34)$ & $17(28,81)$ & $12(20,34$ \\
\hline Yme & $15(25,42)$ & $15(25,42)$ & $12(20,34)$ & $18(30,31)$ & $13(22,03)$ & $17(28,81)$ & $13(22,03)$ & $17(28,81)$ \\
\hline \multicolumn{9}{|c|}{ PNMM lag30m } \\
\hline & \multicolumn{2}{|c|}{$\begin{array}{c}\text { Dia 5 } \\
\mathrm{r}=-0,255\end{array}$} & \multicolumn{2}{|c|}{$\begin{array}{c}\text { Dia } 7 \\
r=-0,406\end{array}$} & \multicolumn{2}{|c|}{$\begin{array}{c}\text { Dia } 9 \\
r=-0,309\end{array}$} & \multicolumn{2}{|c|}{$\begin{array}{c}\text { Dia 13 } \\
\mathrm{r}=-0,430\end{array}$} \\
\hline & Xme & $\mathrm{Xma}$ & Xme & $\mathrm{Xma}$ & Xme & $\mathrm{Xma}$ & Xme & Xma \\
\hline Yma & $14(23,73)$ & $15(25,42)$ & $17(28,81)$ & $12(20,34)$ & $19(32,20)$ & $10(16,95)$ & $21(35,59)$ & $8(13,56)$ \\
\hline Yme & $16(27,12)$ & $14(23,73)$ & $13(22,03)$ & $17(28,81)$ & $11(18,64)$ & $19(32,20)$ & $9(15,25)$ & $21(35,59)$ \\
\hline
\end{tabular}

pico em médio prazo. A correlação $R M S / S P D$ deve tender a zero em prazos de previsão longos, uma vez que a distribuição dos membros do conjunto se aproxima da distribuição climatológica do modelo, a qual não varia dia-a-dia. Assim, o $S P D$ não pode ser utilizado para prever variações no desempenho da previsão por conjunto em longos prazos. De forma análoga, neste estudo, obteve-se o máximo de correlação negativa entre a $C A$ e o $S P D$ para as previsões de médio prazo (7 dias).

De forma geral, os resultados obtidos no experimento lag $30 \mathrm{~m}$ foram comparáveis aos do oper $15 \mathrm{~m}$, o que valida o uso de previsões defasadas na previsão de tempo por conjunto do CPTEC/INPE. Assim, o lag30m constitui uma fonte alternativa de dados para o EPS-CPTEC/INPE, já que usa as previsões provenientes da análise defasada, que contém informações independentes da análise operacional mais recente, com a vantagem de não ter custo computacional adicional.

\section{CONCLUSÕES}

Foi avaliado o impacto da utilização de previsões defasadas nas previsões de tempo por conjunto operacionais do CPTEC/INPE. O conjunto defasado, avaliado neste trabalho, é uma média do conjunto composto pelas 15 previsões perturbadas produzidas pela análise mais recente do dia corrente, e por 15 previsões inicializadas a partir da análise de $12 \mathrm{~h}$ antes.

Diversos experimentos foram avaliados com a finalidade de determinar a melhor estratégia para combinar tais conjuntos de previsões: as previsões determinísticas mais recentes (determ) e as defasadas (lagdet); os conjuntos de previsões mais recente ou operacional (oper $15 \mathrm{~m}$ ) e o defasado (lag $15 \mathrm{~m}$ ); e as combinações dos conjuntos mais recente e defasado sem (lag30m) e com (lag30mw) ponderados pelo erro. A avaliação foi realizada usando índices estatísticos para as variáveis altura geopotencial em $500 \mathrm{hPa}$, temperatura do ar em $850 \mathrm{hPa}$ e pressão ao nível médio do mar, no período de 10 de julho a 10 de setembro de 2007.

A análise dos resultados mostrou que a inclusão de previsões defasadas, como membros do conjunto, contribui para manter e/ou aumentar o desempenho das previsões por conjunto em todas as regiões, HN, TR e HS, e para os três campos avaliados. Além disso, o conjunto composto por membros mais recentes e defasados, apresentou um aumento do espalhamento do conjunto, contribuindo para reduzir a subdispersão do mesmo, com a vantagem de não adicionar custo computacional no processamento operacional das previsões, já que utiliza previsões processadas anteriormente. 
A comparação dos resultados dos pares de experimentos determ e lagdet, e oper $15 \mathrm{~m}$ e lag $15 \mathrm{~m}$, indicou que os desempenhos das previsões defasadas (lagdet) são ligeiramente inferiores às mais recentes (oper $15 \mathrm{~m}$ e determ). Isto indica que a inclusão de termos mais antigos, provinientes de rodada inicializada $12 \mathrm{~h}$ antes do horário mais atual, deve ser ponderada a fim de compensar os erros relativos dos membros provenientes de análises mais antigas. No experimento lag $30 \mathrm{mw}, 15$ membros defasados foram combinados com os 15 mais recentes, considerando uma ponderação pelo inverso da raiz do erro quadrático médio, mediado na região de interesse e médio das 15 previsões mais recentes. Os resultados indicaram que esta ponderação, entretanto, não contribui para um melhor desempenho do conjunto quando comparado ao experimento lag $30 \mathrm{~m}$, em que os 30 membros foram considerados sem ponderação. A não melhoria dos resultados quando os membros mais antigos foram ponderados pode estar associada a maneira com que estão sendo calculados o peso, pelo inverso do RMS médio na região. É possível que este peso tenha que ser calculado para cada ponto de grade e não como médio na área como feito no presente trabalho.

Outro aspecto investigado foi o uso do espalhamento como preditor do desempenho da previsão por conjunto (EPSCPTEC/INPE). Para isto, foram comparadas as previsões geradas a partir dos experimentos oper $15 \mathrm{~m}$, da versão operacional do EPS-CPTEC/INPE, e o lag30m, de previsões defasadas. A análise do diagrama de dispersão e da tabela de contingência das variáveis espalhamento e desempenho, para os prazos de previsão de 5, 7, 9 e 13 dias, mostrou que o espalhamento do conjunto pode ser utilizado como indicador do desempenho do conjunto especialmente para os prazos de 5 e 7 dias. Os resultados indicaram que a correspondência espalhamento pequeno/desempenho alto e, vice-versa, é mais acentuada para o prazo de previsão de 7 dias. Este resultado foi obtido para ambos os experimentos, oper $15 \mathrm{~m}$ e lag $30 \mathrm{~m}$, e não foram encontradas diferenças significativas entre eles, o que valida o uso de previsões defasadas como uma fonte adicional de informações para a previsão por conjunto do CPTEC/INPE.

A utilização de membros defasados pode ser uma fonte efetiva de dados adicionais para a previsão de tempo por conjunto do CPTEC/INPE. A inclusão de previsões defasadas ao conjunto operacional tem um impacto positivo no desempenho das previsões atuais. O experimento lag30m indicou um aumento no espalhamento do conjunto em relação ao oper $15 \mathrm{~m}$ minimizando, assim, a subdispersão do EPSCPTEC/INPE. O desempenho do sistema no HS, usando 30 membros, foi ligeiramente maior comparado ao oper $15 \mathrm{~m}$, com um aumento na correlação de anomalias e redução do $R M S$. Embora esta técnica não resolva totalmente a questão da subdispersão do conjunto, representa uma alternativa simples e de fácil implementação operacional, além de ter baixo custo computacional, já que os membros adicionais são obtidos da rodada anterior a mais recente.

Os resultados deste trabalho ajudam a avaliar e aprimorar o sistema de previsão de tempo por conjunto do CPTEC/INPE. Contribui, também, para apresentar uma forma objetiva de obter informações úteis da previsão de tempo por conjunto, que tem potencial para uso operacional: a relação espalhamento/ desempenho das previsões. Além disso, os resultados apresentados servem como parâmetro para a implementação operacional desta metodologia no EPS do CPTEC/INPE.

\section{REFERÊNCIAS BIBLIOGRÁFICAS}

Barker, T. The relationship between spread and forecast error in extended-range forecast. J. Climate, v. 4, n.7, p. 733742, 1991.

Buizza, R. Potential forecast skill of ensemble prediction and spread and skill distributions of the ECMWF ensemble prediction system. Mon. Wea. Rev, v. 125, n. 1, p. 99-119, 1997.

Coutinho, M. M. Previsão por conjuntos utilizando perturbações baseadas em componentes principais, 1999 . 136p. Dissertação (Mestrado em Meteorologia) - Instituto Nacional de Pesquisas Espaciais, São José dos Campos, 1999.

Dalcher, A.; Kalnay, E. Hoffman, R. Medium Range Lagged Average Forecast. Mon. Wea. Rev., v. 116, n. 2, p. 402415, 1988.

Hoffman, R. N.; Kalnay, E. Lagged average forecasting, an alternative to Monte Carlo forecasting. Tellus, v.35A, n. 2, p.100-118, 1983.

Kanamitsu, M., W. Ebisuzaki, J. Woollen, S-K Yang, J. J. Hnilo, M. Fiorino, G. L. Potter, NCEP-DEO AMIP-II Reanalysis (R-2). Bul. of the Atmos. Met. Soc., p. 1631-1643, 2002.

Lorenz, E.N. Deterministic non-periodic flow. J. Atmos. Sci., v. 20, n. 2, p.130-141, 1963.

Mendonça, A.M; Bonatti, J.P. Experiments with EOF-based perturbation method to ensemble weather forecasting in middle latitudes. In: Proceedings of ICSHMO, 8, 2006, Foz do Iguaçu, Brazil, April 24-28, p. 1829-1832.

Mendonça, A.M.; Bonatti, J.P. Avaliação objetiva do sistema de previsão de tempo global por ensemble do CPTEC/INPE e relação entre o espalhamento e o desempenho do ensemble médio. In: Congresso Brasileiro de Meteorologia, XIII, 29 de agosto a 03 de setembro, 2004, Fortaleza, CE, Anais do XIII Congresso Brasileiro de Meteorologia, Fortaleza, CE, Brasil, 2004.

Molteni, F.; Buizza, R.; Palmer, T.N.; Petroliagis; T. The ECMWF ensemble prediction system: Methodology and 
validation. Quart. J. Roy. Meteor. Soc., v. 122, n. 529, p. 73-119, 1996.

Molteni, F.; Palmer, T.N. A real-time scheme for the prediction of forecast skill. Mon. Wea. Rev., v. 119, n. 2, p. 299-323, 1991.

Murphy, J.M. The impact of the ensemble forecast on predictability. Q. J. R. Meteorol. Soc. v. 114, n. 480, p. 463-493, 1988.

Reynolds, C. A.; Webster, P. J.; Kalnay, E. Random error growth in NMC's global forecasts. Mon. Wea. Rev., v. 122, n. 7, p. 1281-1305, 1994.
Tracton, M. S.; Kalnay, E. Operational ensemble prediction at the National Meteorological Center. Pratical aspects. Wea. Forecasting, v. 8, n. 3, p. 379-398, 1993.

Whitaker, J.S; Loughe, A. The Relationship between Ensemble Spread and Ensemble Mean Skill. Mon.Wea.Rev., v.126, n.12, p. 3292-3302, 1998.

Zhang, Z.; Krishnamurti, T.N. A perturbation method for hurricane ensemble predictions.

Mon.Wea.Rev., v .127, n.4, p.447-469, 1999. 